\title{
Optogenetic probes
}

\author{
Ryohei Yasuda $^{1, *}$ and George J. Augustine ${ }^{1,2, *}$ \\ ${ }^{1}$ Department of Neurobiology, Duke University Medical Center, Box 3209, Durham, NC 27710, USA \\ (*author for correspondence; e-mail: yasuda@neuro.duke.edu) \\ ${ }^{2}$ Program in Neuroscience and Behavioral Disorders, Duke-National University of Singapore \\ Graduate Medical School, 2 Jalan Bukit Merah, Singapore 169547, Singapore ("author for correspondence; \\ e-mail: georgea@neuro.duke.edu)
}

Published online 22 October 2008

(c) Springer Science+Business Media, LLC 2008

We welcome you to our special issue of Brain Cell Biology. The theme of this special issue, Optogenetic probes of neuronal function and circuitry, is intended to provide our readers with a progress report on one of the most exciting areas of contemporary Neuroscience. The goals of this field are to develop and to apply optical probes that either control or detect neuronal activity. Optical techniques make it possible to achieve these goals with high spatiotemporal resolution and in a relatively non-invasive manner. By making these probes genetically encoded, it is possible to obtain great specificity in targeting these probes, so that it is now possible to examine the function of specific synapses and neurons and to determine their contributions to brain circuitry. As you can see from the articles collected in this special issue, these new optogenetic technologies permit many types of experiments that would have been unimaginable only a few years ago and already are providing answers to fundamental questions about the workings of the brain.

In this issue we present articles from many of the leaders in this field. The first group of articles describes the state of the art in developing optogenetic indicators of intracellular signaling:

- Cyclic nucleotides have long been known to play central roles as intracellular second messengers. The article by Pierre Vincent, Nicolas Gervasi, and Jin Zhang describes recent progress in using genetically encoded FRET indicators to dynamically monitor cyclic nucleotide concentrations within neurons.
- Complex intracellular signaling cascades play fundamental roles in most cellular responses. Takeshi Nakamura, Kazuhiro Aoki, and Michiyuki Matsuda describe their recent work using FRET imaging to analyze the signaling network involved in the triggering of neurite outgrowth by the nerve growth factor.

- Optimization of fluorophores is critical for development of sensitive indicators. Hideji Murakoshi, Seok-Jin Lee, and Ryohei Yasuda modify a nonradiative YFP to improve their properties as an acceptor of FRET. When combined with 2photon fluorescence lifetime imaging microscopy, the new fluorophore allows quantitative imaging of protein-protein interactions in single dendritic spines with high sensitivity.

- Aside from development of novel probes, the most important challenge facing the field is development of hardware that is optimal for harnessing the power of modern optogenetic probes. The paper by Takashi Fukano, Satoshi Shimozono, and Atsushi Miyawaki provides a good example of the challenges and rewards associated with development of microscope systems that are optimized for high-speed quantitative $\mathrm{Ca}^{2+}$ imaging using dual-color excitation.

The second group of articles describes progress in developing optogenetic probes of neuronal activity:

- Optical sensors of membrane potential have long promised to provide the ability to simultaneously monitor the electrical activity of many neurons. 
The paper by B. J. Baker, H. Mutoh, D. Dimitrov, W. Akemann, A. Perron, Y. Iwamoto, L. Jin, L. B. Cohen, E. Y. Isacoff, V. A. Pieribone, T. Hughes, and $T$. Knöpfel reviews recent progress in developing genetically encoded fluorescent sensors of membrane potential. Several technical problems that have held back this field have recently been solved, making it likely that the vast promise of these exciting indicators can now be realized.

- Because neuronal excitation is typically associated with activation of voltage-gated $\mathrm{Ca}^{2+}$ channels and attendant elevation of intracellular $\mathrm{Ca}^{2+}$ concentration, fluorescent $\mathrm{Ca}^{2+}$ indicators can provide an alternative means of monitoring neuronal activity. The article by S. Andrew Hires, Lin Tian, and Loren Looger describes several considerations that are important when designing and optimizing genetically encoded $\mathrm{Ca}^{2+}$ indicators and when using these indicators to detect neuronal activity.

- One of the most powerful methods for imaging synaptic activity is to use pHluorins to monitor synaptic vesicle exocytosis and endocytosis. Tomas Fernandez Alfonso and Timothy Ryan describe their use of such technology to discover a new "resting" pool of synaptic vesicles and to describe the temporal and spatial dynamics of this vesicle pool.

- Although excitation of neurons has long been visible through the use of imaging techniques such as voltage-sensitive dyes and fluorescent $\mathrm{Ca}^{2+}$ indicators, synaptic inhibition has largely been invisible to imaging approaches. The paper by Ken Berglund, Wolfram Schleich, Hong Wang, Guoping Feng, William C. Hall, Thomas Kuner, and George Augustine describes the use of the genetically encoded chloride indicator, Clomeleon, to image synaptic inhibition in a variety of brain regions. This approach holds great promise for imaging the spatiotemporal dynamics of inhibitory circuitry in the brain.

The final group of articles considers recent developments in the use of channelrhodopsin-2 and halorhodopsin for optical control of neuronal activity:

- The paper by Philipp Schoenenberger, Åsa Grunditz, Tobias Rose, and Thomas Oertner examines the important issue of spatial resolution when using channelrhodopsin-2 for photostimulation. They show that careful consideration of the light responsiveness of neurons expressing channelrhodopsin-2 allows spatial resolution to be optimized.

- The final two articles-one by Viviana Gradinaru, Kimberly R. Thompson, and Karl Deisseroth and the other by Shengli Zhao, Caterina Cunha, Feng Zhang, Qun Liu, Bernd Gloss, Karl Deisseroth, George Augustine, and Guoping Feng-describe problems in intracellular trafficking of halorhodopsin. Both papers demonstrate that expression of high levels of halorhodopsin causes this light-activated chloride pump to be trapped in the endoplasmic reticulum. Fortunately, these papers also describe several improvements in the amino acid sequence of halorhodopsin that allow this problem to be circumvented. The paper by Zhao et al. also describes the first transgenic mouse lines that allow halorhodopsin-based photoinhibition of neuronal activity.

As you can see from this impressive list, this issue has many outstanding articles and surely will be a significant landmark in the development of the nascent field of optogenetics.

We will close by thanking each author for their excellent contributions to this very special issue. We hope that $B C B$ readers will enjoy reading this issue as much as we have enjoyed assembling it.

\section{Ryohei Yasuda \\ Guest Editor}

George Augustine

Editor in Chief

Brain Cell Biology 